\title{
GREEN FUNCTION FOR GRADIENT PERTURBATION OF UNIMODAL LÉVY PROCESSES
}

\author{
BY
}

TOMASZ GRZYWNY* (WroceaW), TOMASZ JAKU BOWS K I** (WrocŁaW), AND GRZEGORZ ŻURE K (WROCEAW)

\begin{abstract}
We prove that the Green function of a generator of isotropic unimodal Lévy processes with the weak lower scaling order greater than one and the Green function of its gradient perturbations are comparable for bounded smooth open sets if the drift function is from an appropriate Kato class.
\end{abstract}

2010 AMS Mathematics Subject Classification: Primary: 47A55, 60J50, 60J75, 47G20; Secondary: 60J35.

Key words and phrases: Unimodal Lévy process, heat kernel, smooth domain, Green function, gradient perturbation.

\section{INTRODUCTION}

Let $X_{t}$ be a pure-jump isotropic unimodal Lévy process on $\mathbb{R}^{d}, d \geqslant 2$. That is, $X_{t}$ is a Lévy process with a rotationally invariant and radially non-increasing density function $p_{t}(x)$ on $\mathbb{R}^{d} \backslash\{0\}$. The characteristic exponent of $\left\{X_{t}\right\}$ equals

$$
\psi(x)=\int_{\mathbb{R}^{d}}(1-\cos (x \cdot z)) \nu(d z), \quad x \in \mathbb{R}^{d},
$$

where $\nu$ is a Lévy measure, i.e., $\int_{\mathbb{R}^{d}}\left(1 \wedge|z|^{2}\right) \nu(d z)<\infty$. For general information on unimodal processes, we refer the reader to [3], [15], [3]]. One of the primary examples of the mentioned class of processes is the isotropic $\alpha$-stable Lévy process having the fractional Laplacian $\Delta^{\alpha / 2}$ as a generator.

Perturbations of $\Delta^{\alpha / 2}$ by the first order operators are currently widely studied by many authors from various points of view, see [5]-[8], [10], [114], [18], [19], [22], [24]-[26], [28], [29]. In a recent paper [6] the authors studied the Green function of $\Delta^{\alpha / 2}+b(x) \cdot \nabla$ in bounded $C^{1,1}$ domains. Here $b$ is a vector field

\footnotetext{
* Tomasz Grzywny was supported by the Alexander von Humboldt Foundation.
}

** Tomasz Jakubowski was partially supported by grant MNiSW IP2012 018472. 
from the Kato class $\mathcal{K}_{d}^{\alpha-1}$. It was shown that the Green function of the original process is comparable with the Green function of the perturbed process for any bounded $C^{1,1}$ open set. In this paper we generalize the result of [6] to the case of isotropic unimodal Lévy processes. Let

$$
\mathcal{L} f(x)=\int_{\mathbb{R}^{d}}\left(f(x+z)-f(x)-\mathbb{1}_{|z|<1}(z \cdot \nabla f(x))\right) \nu(d z), \quad f \in C_{b}^{2}\left(\mathbb{R}^{d}\right),
$$

be a generator of the process $X_{t}$. We will consider a non-empty bounded open $C^{1,1}$ set $D$ and the Green function $G_{D}$ for $\mathcal{L}$. Now, let $\tilde{G}_{D}(x, y)$ be a Green function for

$$
\tilde{\mathcal{L}}=\mathcal{L}+b(x) \cdot \nabla
$$

where $b$ is a function from the Kato class $\mathcal{K}_{d}^{\nabla}$ (see Section 2 for details). Our main result is

THEOREM 1.1. Let $d \geqslant 2, b \in \mathcal{K}_{d}^{\nabla}$, and let $D \subset \mathbb{R}^{d}$ be a bounded $C^{1,1}$ open set. We assume that the characteristic exponent

$$
\psi \in \operatorname{WLSC}(\underline{\alpha}, 0, \underline{c}) \cap \operatorname{WLSC}\left(\underline{\alpha}_{1}, 1, \underline{c}_{1}\right) \cap \operatorname{WUSC}(\bar{\alpha}, 0, \bar{C}), \quad \text { where } \underline{\alpha}_{1}>1,
$$

$$
\left|\nabla_{x} G_{D}(x, y)\right| \leqslant C_{0} \frac{G_{D}(x, y)}{|x-y| \wedge \delta_{D}(x) \wedge 1} .
$$

Then, there exists a constant $C$ such that, for $x, y \in D$,

$$
C^{-1} G_{D}(x, y) \leqslant \tilde{G}_{D}(x, y) \leqslant C G_{D}(x, y) .
$$

Here WLSC and WUSC are the classes of functions satisfying a weak lower and a weak upper scaling condition, respectively (see Section 2). The condition (IL.2) is satisfied for a wide class of processes. For example, (11.2) holds under a mild assumption on a density of the Lévy measure, which is satisfied for any subordinate Brownion motion (see Lemma [3.2) (see also [12], Theorem 1.4).

Generally, we follow the approach of [6]. Since some proofs are almost identical to the ones from [6], we omit them. The main tool, we use in this paper, is the Duhamel (perturbation) formula (see Theorem B.T). We note that this result cannot be obtained directly in the same way as the perturbation formula for fractional Laplacian (see [6], Lemma 12). One of the other difficulties in this paper is that we do not have the explicit formula for the potential kernel $G(x)$ of $X_{t}$. Moreover, for stable process, $\psi(\xi)=|\xi|^{\alpha}$, which gives a nice scaling of some main objects. Here, we have only a weak scaling, but it is sufficient for our purpose, although it makes the calculations a little harder. For example, in the estimates of the Green function a factor $V\left(\delta_{D}(x)\right)$ appears. For stable process, $V(r)=r^{\alpha / 2}$, and if $y$ is such that $\delta_{D}(y)=\lambda \delta_{D}(x)$, then $V\left(\delta_{D}(y)\right)=\lambda^{\alpha / 2} V\left(\delta_{D}(x)\right)$. For the general unimodal process, $V$ satisfies the weak scaling condition, and we can only estimate $V\left(\delta_{D}(y)\right)$. 
The paper is organized as follows. In Section 2, we give the definitions of the processes $X$ and $\tilde{X}$ and present their basic properties. In Section 3, we introduce Green functions of $X$ and $\tilde{X}$. Lastly, in Section 4, we prove Theorem L.].

When we write $f(x) \approx g(x)$, we mean that there is a number $0<C<\infty$ independent of $x$, i.e., a constant such that for every $x$ we have $C^{1} f(x) \leqslant g(x) \leqslant$ $C f(x)$. The notation $C=C\left(a_{1}, a_{2}, \ldots, a_{n}\right)$ means that $C$ is a constant which depends only on $a_{1}, a_{2}, \ldots, a_{n}$. We use the convention that constants denoted by capital letters do not change throughout the paper. For a radial function $f: \mathbb{R}^{d} \rightarrow$ $[0, \infty)$ we shall often write $f(r)=f(x)$ for any $x \in \mathbb{R}^{d}$ with $|x|=r$.

\section{PRELIMINARIES}

In what follows, $\mathbb{R}^{d}$ denotes the Euclidean space of dimension $d \geqslant 2$, $d y$ stands for the Lebesgue measure on $\mathbb{R}^{d}$. Without further mention we will only consider Borelian sets, measures and functions in $\mathbb{R}^{d}$. As usual, we write $a \wedge b=$ $\min (a, b)$ and $a \vee b=\max (a, b)$. By $x \cdot y$ we denote the Euclidean scalar product of $x, y \in \mathbb{R}^{d}$. We let $B(x, r)=\left\{y \in \mathbb{R}^{d}:|x-y|<r\right\}$. For $D \subset \mathbb{R}^{d}$, the distance to the complement of $D$ will be denoted by

$$
\delta_{D}(x)=\operatorname{dist}\left(x, D^{c}\right) .
$$

DEFINITION 2.1. Let $\underline{\theta} \in[0, \infty)$, and $\phi$ be a non-negative non-zero function on $(0, \infty)$. We say that $\phi$ satisfies the weak lower scaling condition (at infinity) if there are numbers $\underline{\alpha}>0$ and $\underline{c} \in(0,1]$ such that

$$
\phi(\lambda \theta) \geqslant \underline{c} \lambda^{\underline{\alpha}} \phi(\theta) \quad \text { for } \lambda \geqslant 1, \theta>\underline{\theta} .
$$

In short, we say that $\phi$ satisfies $\operatorname{WLSC}(\underline{\alpha}, \underline{\theta}, \underline{c})$ and write $\phi \in \operatorname{WLSC}(\underline{\alpha}, \underline{\theta}, \underline{c})$. If $\phi \in \operatorname{WLSC}(\underline{\alpha}, 0, \underline{c})$, then we say that $\phi$ satisfies the global weak lower scaling condition.

We consider similarly $\bar{\theta} \in[0, \infty)$. The weak upper scaling condition holds if there are numbers $\bar{\alpha}<2$ and $\bar{C} \in[1, \infty)$ such that

$$
\phi(\lambda \theta) \leqslant \bar{C} \lambda^{\bar{\alpha}} \phi(\theta) \quad \text { for } \lambda \geqslant 1, \theta>\bar{\theta} .
$$

In short, $\phi \in \operatorname{WUSC}(\bar{\alpha}, \bar{\theta}, \bar{C})$. For global weak upper scaling we require that $\bar{\theta}=0$ in (2.2).

Throughout the paper, $X_{t}$ will be the pure-jump isotropic unimodal Lévy process on $\mathbb{R}^{d}$. The Lévy measure $\nu$ of $X_{t}$ is radially symmetric and non-increasing, so it admits the radial density $\nu$, i.e., $\nu(d x)=\nu(|x|) d x$. Hence the characteristic exponent $\psi$ of $X_{t}$ is radial as well. We assume that (see Theorem ㄴ.J)

$$
\begin{aligned}
& \psi \in \operatorname{WLSC}(\underline{\alpha}, 0, \underline{c}) \cap \operatorname{WUSC}(\bar{\alpha}, 0, \bar{C}), \\
& \psi \in \operatorname{WLSC}\left(\underline{\alpha}_{1}, 1, \underline{c}_{1}\right) \quad \text { for some } \underline{\alpha}_{1}>1 .
\end{aligned}
$$


Following [27], we define

$$
h(r)=\int_{\mathbb{R}^{d}}\left(1 \wedge \frac{|x|^{2}}{r^{2}}\right) \nu(|x|) d x, \quad r>0 .
$$

Let us notice that

$$
h(\lambda r) \leqslant h(r) \leqslant \lambda^{2} h(\lambda r), \quad \lambda>1 .
$$

Moreover, by [3] , Lemma 1 and (6),

$$
2^{-1} \psi(1 / r) \leqslant h(r) \leqslant C_{1} \psi(1 / r) .
$$

In fact, we may write $C_{1}=d \pi^{2} / 2$, but it will be more convenient to write this constant as $C_{1}$. We define the function $V$ as follows:

$$
V(0)=0 \quad \text { and } \quad V(r)=1 / \sqrt{h(r)}, \quad r>0 .
$$

Since $h(r)$ is non-increasing, $V$ is non-decreasing. We have

$$
V(r) \leqslant V(\lambda r) \leqslant \lambda V(r), \quad r \geqslant 0, \lambda>1 .
$$

By weak scaling properties of $\psi$ and the property $h(r) \approx \psi(1 / r)$, we get

$$
\begin{gathered}
\left(\frac{\underline{c}}{2 C_{1}}\right)^{1 / 2} \lambda^{\underline{\alpha} / 2} \leqslant \frac{V(\lambda r)}{V(r)} \leqslant\left(2 \bar{C} C_{1}\right)^{1 / 2} \lambda^{\bar{\alpha} / 2}, \quad r>0, \lambda>1, \\
\frac{V(\eta r)}{V(r)} \leqslant\left(\frac{2 C_{1}}{\underline{c}_{1}}\right)^{1 / 2} \eta^{\underline{\alpha}_{1} / 2}, \quad \eta<1, r<1 .
\end{gathered}
$$

Therefore, $V \in \operatorname{WLSC}\left(\underline{\alpha} / 2,0, \sqrt{\underline{c} /\left(2 C_{1}\right)}\right) \cap \operatorname{WUSC}\left(\bar{\alpha} / 2,0, \sqrt{2 \bar{C} C_{1}}\right)$.

REMARK 2.1. The threshold $(0,1)$ in the scaling of $V$ in (2.7) may be replaced by any bounded interval at the expense of constant $\sqrt{2 C_{1} / \underline{c}_{1}}$ (see [3], Section 3), i.e., for any $R>1$, there is a constant $c$ such that

$$
\frac{V(\eta r)}{V(r)} \leqslant c \eta^{\underline{\alpha}_{1} / 2}, \quad \eta<1, r<R .
$$

The global weak lower scaling condition (assumption (2.3)) implies that $p_{t}(x)$ is jointly continuous on $(0, \infty) \times \mathbb{R}^{d}\left(e^{-t \psi} \in L^{1}\left(\mathbb{R}^{d}\right)\right.$ ) and (see [4], Lemma 1.5)

$$
\begin{aligned}
& p_{t}(x) \approx\left[V^{-1}(\sqrt{t})\right]^{-d} \wedge \frac{t}{V^{2}(|x|)|x|^{d}}, \\
& \nu(x) \approx \frac{1}{V^{2}(|x|)|x|^{d}} .
\end{aligned}
$$

Analogously to $\alpha$-stable processes we define the Kato class for gradient perturbations. 
DEFINITION 2.2. We say that a vector field $b: \mathbb{R}^{d} \rightarrow \mathbb{R}^{d}$ belongs to the Kato class $\mathcal{K}_{d}^{\nabla}$ if

$$
\lim _{r \rightarrow 0^{+}} \sup _{x \in \mathbb{R}^{d}} \int_{B(x, r)} \frac{V^{2}(|x-z|)}{|x-z|^{d+1}}|b(z)| d z=0 .
$$

REMARK 2.2. We note that $L^{\infty}\left(\mathbb{R}^{d}\right) \subset \mathcal{K}_{d}^{\nabla}$.

Let us put

$$
p(t, x, y)=p_{t}(y-x) .
$$

By [16], Theorem B.5, we have

$$
\left|\nabla_{x} p(t, x, y)\right| \leqslant c \frac{1}{V^{-1}(\sqrt{t})} p(t, x, y), \quad t>0, x, y \in \mathbb{R}^{d} .
$$

Let $b \in \mathcal{K}_{d}^{\nabla}$. Following [5] and [20], for $t>0$ and $x, y \in \mathbb{R}^{d}$, we recursively define

$$
\begin{gathered}
p_{0}(t, x, y)=p(t, x, y), \\
p_{n}(t, x, y)=\int_{0}^{t} \int_{\mathbb{R}^{d}} p_{n-1}(t-s, x, z) b(z) \cdot \nabla_{z} p(s, z, y) d z d s, \quad n \geqslant 1,
\end{gathered}
$$

and we let

$$
\tilde{p}=\sum_{n=0}^{\infty} p_{n}
$$

By [20], Theorem 1.1, the series converges to a probability transition density function, and

$$
c_{T}^{-1} p(t, x, y) \leqslant \tilde{p}(t, x, y) \leqslant c_{T} p(t, x, y), \quad x, y \in \mathbb{R}^{d}, 0<t<T,
$$

where $c_{T} \rightarrow 1$ if $T \rightarrow 0$, see [20], Theorem 3 . Moreover, one can prove that $\tilde{p}$ is jointly continuous on $(0, \infty) \times \mathbb{R}^{d} \times \mathbb{R}^{d}$ (see [5], Corollary 19).

We consider the time-homogeneous transition probabilities

$$
P_{t}(x, A)=\int_{A} p(t, x, y) d y, \quad \tilde{P}_{t}(x, A)=\int_{A} \tilde{p}(t, x, y) d y,
$$

$t>0, x \in \mathbb{R}^{d}, A \subset \mathbb{R}^{d}$. By Kolmogorov's and Dynkin-Kinney's theorems the transition probabilities $P_{t}$ and $\tilde{P}_{t}$ define, in a usual way, Markov probability measures $\left\{\mathbb{P}^{x}, \tilde{\mathbb{P}}^{x}, x \in \mathbb{R}^{d}\right\}$ on the space $\Omega$ of the right-continuous and left-limited functions $\omega:[0, \infty) \rightarrow \mathbb{R}^{d}$. We let $\mathbb{E}^{x}, \tilde{\mathbb{E}}^{x}$ be the corresponding expectations. We will denote by $X=\left\{X_{t}\right\}_{t \geqslant 0}$ the canonical process on $\Omega, X_{t}(\omega)=\omega(t)$. Hence,

$$
\mathbb{P}^{x}\left(X_{t} \in B\right)=\int_{B} p(t, x, y) d y, \quad \tilde{\mathbb{P}}^{x}\left(X_{t} \in B\right)=\int_{B} \tilde{p}(t, x, y) d y .
$$


For any open set $D$ we define the first exit time of the process $X_{t}$ from $D$,

$$
\tau_{D}=\inf \left\{t>0: X_{t} \notin D\right\} .
$$

Now, by the usual Hunt's formula, we define the transition density of the process killed when leaving $D$ (see [1]], [13], [4]):

$$
p_{D}(t, x, y)=p(t, x, y)-\mathbb{E}^{x}\left[\tau_{D}<t ; p\left(t-\tau_{D}, X_{\tau_{D}}, y\right)\right], \quad t>0, x, y \in \mathbb{R}^{d} .
$$

We briefly recall some well-known properties of $p_{D}$ (see [4]). The function $p_{D}$ satisfies the Chapman-Kolmogorov equations

$$
\int_{\mathbb{R}^{d}} p_{D}(s, x, z) p_{D}(t, z, y) d z=p_{D}(s+t, x, y), \quad s, t>0, x, y \in \mathbb{R}^{d} .
$$

Furthermore, $p_{D}$ is jointly continuous (compare Lemma [2.3]) when $t \neq 0$, and we have

$$
0 \leqslant p_{D}(t, x, y)=p_{D}(t, y, x) \leqslant p(t, x, y)
$$

In particular,

$$
\int_{\mathbb{R}^{d}} p_{D}(t, x, y) d y \leqslant 1
$$

By Blumenthal's zero-one law, radial symmetry of $p_{t}$ and $C^{1,1}$ geometry of the boundary $\partial D$, we have $\mathbb{P}^{x}\left(\tau_{D}=0\right)=1$ for every $x \in D^{c}$. In particular, $p_{D}(t, x, y)$ $=0$ if $x \in D^{c}$ or $y \in D^{c}$. By the strong Markov property,

$$
\mathbb{E}^{x}\left[t<\tau_{D} ; f\left(X_{t}\right)\right]=\int_{\mathbb{R}^{d}} f(y) p_{D}(t, x, y) d y, \quad t>0, x \in \mathbb{R}^{d},
$$

for functions $f \geqslant 0$.

For $s \in \mathbb{R}, x \in \mathbb{R}^{d}$, and $\phi \in C_{c}^{\infty}(\mathbb{R} \times D)$, we have (see [2], Remark 4.2, and [6], the proof of Lemma 5)

$$
\int_{s}^{\infty} \int_{D} p_{D}(u-s, x, z)\left[\partial_{u} \phi(u, z)+\mathcal{L}_{z} \phi(u, z)\right] d z d u=-\phi(s, x),
$$

which justifies calling $p_{D}$ the Dirichlet heat kernel of $\mathcal{L}$ on $D$.

In a similar way, we define an analogous object for the process $\tilde{X}$. Let $\tilde{\tau}_{D}=$ $\inf \left\{t>0: \tilde{X}_{t} \notin D\right\}$. By Hunt's formula,

$$
\tilde{p}_{D}(t, x, y)=\tilde{p}(t, x, y)-\tilde{\mathbb{E}}^{x}\left[\tau_{D}<t ; \tilde{p}\left(t-\tau_{D}, X_{\tau_{D}}, y\right)\right] .
$$

Except symmetry, $\tilde{p}_{D}$ has analogous properties to $p_{D}$, i.e., the Chapman-Kolmogorov equation holds,

$$
\int_{\mathbb{R}^{d}} \tilde{p}_{D}(s, x, z) \tilde{p}_{D}(t, z, y) d z=\tilde{p}_{D}(s+t, x, y), \quad s, t>0, x, y \in \mathbb{R}^{d},
$$

and $0 \leqslant \tilde{p}_{D}(t, x, y) \leqslant \tilde{p}(t, x, y)$. Now, we will prove that $\tilde{p}_{D}$ is jointly continuous on $(0, \infty) \times D \times D$. First, we need two preparatory lemmas. 
LeMmA 2.1. Let $\delta>0$. Then $M_{\delta}:=\sup _{t>0,|x-y| \geqslant \delta} \tilde{p}(t, x, y)<\infty$.

Pr o of. By (2.13) and [3], Corollary 7 , for $t \leqslant 1$,

$$
\tilde{p}(t, x, y) \leqslant c \frac{t}{V^{2}(|x-y|)|x-y|^{d}} .
$$

Hence,

$$
\sup _{0<t \leqslant 1,|x-y| \geqslant \delta} \tilde{p}(t, x, y) \leqslant \frac{c}{V^{2}(\delta) \delta^{d}} .
$$

Furthermore, by the semigroup property, for $t>1$,

$$
\tilde{p}(t, x, y) \leqslant c \int_{\mathbb{R}^{d}} \tilde{p}(t-1, x, z) p(1, z-y) d z \leqslant c p(1,0),
$$

which implies

$$
M_{\delta} \leqslant c \max \left\{\left(V(\delta) \delta^{d}\right)^{-1}, p(1,0)\right\}<\infty
$$

LEMMA 2.2. Let $\delta>0$. Then

$$
\begin{array}{r}
\lim _{s \rightarrow 0^{+}} \sup _{t \leqslant s, x \in \mathbb{R}^{d}} \tilde{\mathbb{P}}^{x}\left(\left|X_{t}-X_{0}\right| \geqslant \delta\right)=0, \\
\lim _{s \rightarrow 0^{+}} \sup _{x \in \mathbb{R}^{d}} \tilde{\mathbb{P}}^{x}\left(\tau_{B(x, \delta)} \leqslant s\right)=0 .
\end{array}
$$

Pro of. Let $s \leqslant 1$ and $t \leqslant s$. By (2.13]) and [3], Corollary 6 ,

$$
\tilde{\mathbb{P}}^{x}\left(\left|X_{t}-X_{0}\right| \geqslant \delta\right) \leqslant c_{1} \int_{B_{\delta}^{c}} p(t, y) d y \leqslant c \frac{t}{V^{2}(\delta)} \leqslant c(\delta) s .
$$

Hence, we obtain (2.18). The equality $(2.19)$ is a consequence of $(2.18)$ and the strong Markov property (see [10], the proof of Lemma 3.1).

Although, in this paper, we consider only bounded sets, the following lemma also holds for unbounded domains. To obtain it we use standard arguments (e.g., [13], Theorem 2.4).

LEMMA 2.3. $\tilde{p}_{D}$ is jointly continuous on $(0, \infty) \times D \times D$.

Proof. Let $0<\delta<r, D^{\delta}=\left\{y \in D: \delta_{D}(y) \geqslant \delta\right\}$ and $D_{r}^{\delta}=D^{\delta} \cap B_{r}$. Generally, $\delta$ is close to zero and $r$ is large. We assume that $(t, x, y) \in[\delta, r] \times$ $D^{\delta} \times D_{r}^{\delta}$. We denote by

$$
\tilde{r}_{D}(t, x, y)=\tilde{\mathbb{E}}^{x}\left[\tilde{p}\left(t-\tau_{D}, X_{\tau_{D}}, y\right), \tau_{D}<t\right]
$$

the killing measure of $\tilde{X}$. Hence,

$$
\tilde{p}_{D}(t, x, y)=\tilde{p}(t, x, y)-\tilde{r}_{D}(t, x, y) .
$$


Let $s<\delta / 2$,

$$
h_{s}(t, x, y)=\tilde{\mathbb{E}}^{x}\left[\tilde{p}\left(t-s-\tau_{D}, X_{\tau_{D}}, y\right), \tau_{D}<t-s\right],
$$

and $\phi_{s}(t, x, y)=\tilde{\mathbb{E}}^{x} h_{s}\left(t, X_{s}, y\right)$. By the Markov property,

$$
\begin{aligned}
& \tilde{r}_{D}(t, x, y)-\phi_{s}(t, x, y)=\tilde{\mathbb{E}}^{x}\left[\tilde{p}\left(t-\tau_{D}, X_{\tau_{D}}, y\right), \tau_{D} \leqslant s\right] \\
&-\tilde{\mathbb{E}}^{x}\left[\tau_{D} \leqslant s, \tilde{\mathbb{E}}^{X}\left[\tilde{p}\left(t-s-\tau_{D}, X_{\tau_{D}}, y\right), \tau_{D}<t-s\right]\right] .
\end{aligned}
$$

\section{By Lemma 2.1],}

$$
\left|\tilde{r}_{D}(t, x, y)-\phi_{s}(t, x, y)\right| \leqslant 2 M_{\delta} \tilde{\mathbb{P}}^{x}\left(\tau_{D} \leqslant s\right) \leqslant 2 M_{\delta} \sup _{z \in \mathbb{R}^{d}} \tilde{\mathbb{P}}^{z}\left(\tau_{B(z, \delta)} \leqslant s\right) .
$$

Hence, by (2.19), it is enough to prove the continuity of $\phi_{s}$ on $[\delta, r] \times D^{\delta} \times D_{r}^{\delta}$ for $0<s<\delta / 2$.

First, we prove the equicontinuity of $h_{s}(\cdot, z, \cdot)$ on $[\delta, r] \times D_{r}^{\delta}$ for $z \in \mathbb{R}^{d}$. Fix $\varepsilon>0$. By (2.13) and (2.9), there is $0<\lambda \leqslant \delta / 4$ such that, for $w \in D^{c}, v \in D^{\delta}$ and $u \leqslant \lambda$,

$$
\tilde{p}(u, w, v) \leqslant c \frac{\lambda}{V^{2}(\delta) \delta^{d}}<\varepsilon .
$$

Next, by the semigroup property, ([2.9) and ([2.13) , there is $R \geqslant 2 r$ such that, for $w \in B_{R}^{c}, v \in B_{r}$ and $u \leqslant r$,

$$
\tilde{p}(u, w, v) \leqslant \frac{c r}{V^{2}(R / 2) R^{d}}<\varepsilon .
$$

Now, we divide $h_{s}$ into three parts and treat them separately,

$$
h_{s}(t, z, y)=J_{1}(t, z, y)+J_{2}(t, z, y)+J_{3}(t, z, y),
$$

where

$$
\begin{aligned}
& J_{1}(t, z, y)=\tilde{\mathbb{E}}^{z}\left[\tilde{p}\left(t-s-\tau_{D}, X_{\tau_{D}}, y\right), \tau_{D}<t-s-\lambda, X_{\tau_{D}} \in B_{R}\right], \\
& J_{2}(t, z, y)=\tilde{\mathbb{E}}^{z}\left[\tilde{p}\left(t-s-\tau_{D}, X_{\tau_{D}}, y\right), t-s-\lambda \leqslant \tau_{D}<t-s\right], \\
& J_{3}(t, z, y)=\tilde{\mathbb{E}}^{z}\left[\tilde{p}\left(t-s-\tau_{D}, X_{\tau_{D}}, y\right), \tau_{D}<t-s-\lambda, X_{\tau_{D}} \in B_{R}^{c}\right] .
\end{aligned}
$$

By (2.2]) and (2.22),

$$
J_{2}(t, z, y)+J_{3}(t, z, y)<2 \varepsilon, \quad z \in \mathbb{R}^{d},(t, y) \in[\delta, r] \times D_{r}^{\delta} .
$$

Since $\tilde{p}(\cdot, \cdot, \cdot)$ is continuous on $(0, \infty) \times \mathbb{R}^{d} \times \mathbb{R}^{d}$, it is uniformly continuous on $[\lambda / 2, r] \times B_{R} \times B_{r}$. Hence, there is $0<\varepsilon_{1} \leqslant \lambda / 2$ such that, for $(u, w),\left(u_{0}, w_{0}\right) \in$ $[\lambda / 2, r] \times D_{r}^{\delta}$ and $w \in B_{R}$,

(2.24) $\left|\tilde{p}(u, v, w)-\tilde{p}\left(u_{0}, v, w_{0}\right)\right|<\varepsilon \quad$ if $\left|(u, w)-\left(u_{0}, w_{0}\right)\right|<\varepsilon_{1}, v \in \mathbb{R}^{d}$. 
Assume that $\left(t_{0}, y_{0}\right) \in[\delta, r] \times D_{r}^{\delta}$ and $t_{0} \leqslant t$. Then

$$
\begin{aligned}
J_{1}\left(t_{0}, z, y_{0}\right)= & \tilde{\mathbb{E}}^{z}\left[\tilde{p}\left(t_{0}-s-\tau_{D}, X_{\tau_{D}}, y_{0}\right), \tau_{D}<t-s-\lambda, X_{\tau_{D}} \in B_{R}\right] \\
& -\tilde{\mathbb{E}}^{z}\left[\tilde{p}\left(t_{0}-s-\tau_{D}, X_{\tau_{D}}, y_{0}\right), t_{0} \leqslant \tau_{D}+s+\lambda<t, X_{\tau_{D}} \in B_{R}\right] .
\end{aligned}
$$

This, (2.2]) and (2.24) imply, for $(t, y),\left(t_{0}, y_{0}\right) \in[\delta, r] \times D_{r}^{\delta}$,

$$
\sup _{z \in \mathbb{R}^{d}}\left|J_{1}(t, z, y)-J_{1}\left(t_{0}, z, y_{0}\right)\right|<2 \varepsilon \quad \text { if }\left|(t, y)-\left(t_{0}, y_{0}\right)\right|<\varepsilon_{1}, z \in \mathbb{R}^{d} .
$$

Combining (2.23) with (2.25) gives the equicontinuity of $h_{s}(\cdot, z, \cdot)$ on $[\delta, r] \times D_{r}^{\delta}$ for $z \in \mathbb{R}^{d}$.

This implies the equicontinuity of $\phi_{s}(\cdot, z, \cdot)$ on $[\delta, r] \times D_{r}^{\delta}$ for $z \in \mathbb{R}^{d}$. Since $\tilde{P}_{s}$ is strong Feller, $\phi_{s}(t, \cdot, y)$ is continuous on $\mathbb{R}^{d}$. Therefore, $\phi_{s}(\cdot, \cdot, \cdot)$ is jointly continuous on $[\delta, r] \times \mathbb{R}^{d} \times D_{r}^{\delta}$. By $(2.20)$ and (2.19) $), r_{D}(\cdot, \cdot, \cdot)$ is jointly continuous on $[\delta, r] \times D_{r}^{\delta} \times D_{r}^{\delta}$, which implies continuity on $(0, \infty) \times D \times D$. Since $\tilde{p}$ is jointly continuous, $\tilde{p}_{D}$ is jointly continuous on $(0, \infty) \times D \times D$.

By similar calculations to those in [20], Theorem 2, one can prove that $\tilde{p}$ is the fundamental solutions for $\tilde{\mathcal{L}}$.

Lemma 2.4. For $s>0, x \in D$ and $\phi \in C_{c}^{\infty}((0, \infty) \times D)$, we have

$$
\int_{s}^{\infty} \int_{D} \tilde{p}_{D}(u-s, x, z)\left(\partial_{u}+\tilde{\mathcal{L}}\right) \phi(u, z) d z d u=-\phi(s, x) .
$$

\section{GREEN FUNCTIONS}

In this section we define and prove some properties of the Green functions of $\mathcal{L}$ and $\tilde{\mathcal{L}}$.

\subsection{Green function of $\mathcal{L}$.}

DEFINITION 3.1. A non-empty open set $D \subset \mathbb{R}^{d}$ is of class $C^{1,1}$ at scale $r>0$ if for every $Q \in \partial D$ there are balls $B\left(x^{\prime}, r\right) \subset D$ and $B\left(x^{\prime \prime}, r\right) \subset D^{c}$ tangent at $Q$.

If $D$ is $C^{1,1}$ at some unspecified scale (hence also at all smaller scales), then we simply say that $D$ is $C^{1,1}$. The localization radius,

$$
r_{0}=r_{0}(D)=\sup \left\{r: D \text { is } C^{1,1} \text { at scale } r\right\},
$$

refers to the local geometry of $D$, while the diameter,

$$
\operatorname{diam}(D)=\sup \{|x-y|: x, y \in D\},
$$

refers to the global geometry of $D$. The ratio $\operatorname{diam}(D) / r_{0}(D) \geqslant 2$ will be called the distortion of $D$. We can localize each $C^{1,1}$ open set as follows (see [5], Lemma 1): 
LEMmA 3.1. There exists $\kappa>0$ such that if $D$ is $C^{1,1}$ at scale $r$ and $Q \in \partial D$, then there is a $C^{1,1}$ domain $F \subset D$ with $r_{0}(F)>\kappa r$, $\operatorname{diam}(F)<2 r$ and

$$
D \cap B(Q, r / 4)=F \cap B(Q, r / 4) .
$$

We will write $F=F(z, r)$, and note that the distortion of $F$ is at most $2 / \kappa$, an absolute constant.

In what follows $D$ will be a non-empty bounded $C^{1,1}$ open set in $\mathbb{R}^{d}$. We note that such $D$ may be disconnected, but then it may only have a finite number of connected components at a positive distance from each other.

Definition 3.2. We say that a function $h$ is $\mathcal{L}$-harmonic in the open set $D$ if for every $U$ such that $\bar{U} \subset D$ we have

$$
h(x)=\mathbb{E}^{x} h\left(X_{\tau_{U}}\right), \quad x \in \mathbb{R}^{d} .
$$

We define the Green function of $\mathcal{L}$ for $D$,

$$
G_{D}(x, y)=\int_{0}^{\infty} p_{D}(t, x, y) d t, \quad x, y \in \mathbb{R}^{d} .
$$

We briefly recall some basic properties of $G_{D}(x, y)$ (see [4] for details). For $x \in$ $D^{c}$ or $y \in D^{c}, G_{D}(x, y)=0$. The Green function $G_{D}(x, y)$ is symmetric, continuous for $x \neq y$, and $G_{D}(x, x)=\infty$ for $x \in D$. Furthermore, $G_{D}(\cdot, y)$ is $\mathcal{L}$ harmonic in $D \backslash\{y\}$ for every $y \in D$. We also have

LEMmA 3.2. Let $-\nu^{\prime}(r) / r$ be non-increasing. Then (IL.2) holds.

Proof. Since $G_{D}(\cdot, y)$ is $\mathcal{L}$-harmonic on $D \backslash B(y, r)$ for small $r>0$, by (2.10) and by Theorem 1.1 and Proposition 1.3 in [23], we have

$$
\left|\nabla_{x} G_{D}(x, y)\right| \leqslant c \frac{G_{D}(x, y)}{\delta_{D \backslash B(y,|x-y| / 2)}(x)} \leqslant 2 c \frac{G_{D}(x, y)}{|x-y| \wedge \delta_{D}(x) \wedge 1} .
$$

The Green operator of $\mathcal{L}$ for $D$ is

$$
G_{D} f(x)=\mathbb{E}^{x} \int_{0}^{\tau_{D}} f\left(X_{t}\right) d t=\int_{\mathbb{R}^{d}} G_{D}(x, y) f(y) d y, \quad x \in \mathbb{R}^{d},
$$

and we have

$$
G_{D}(\mathcal{L} \phi)(x)=\int_{D} G_{D}(x, y) \mathcal{L} \phi(y) d y=-\phi(x), \quad x \in \mathbb{R}^{d}, \phi \in C_{c}^{\infty}(D) .
$$

By the Ikeda-Watanabe formula [17], the $\mathbb{P}^{x}$-distribution of $X_{\tau_{D}}$ has a density function, called the Poisson kernel and defined as

$$
P_{D}(x, z)=\int_{D} G_{D}(x, y) \nu(z-y) d y, \quad x \in D, z \in(\bar{D})^{c} .
$$


Hence,

$$
\mathbb{P}^{x}\left(X_{\tau_{D}} \in B\right)=\int_{B} P_{D}(x, z) d z, \quad B \subset(\bar{D})^{c} .
$$

Because of the $C^{1,1}$ geometry of $D, \mathbb{P}^{x}\left(X_{\tau_{D}} \in \partial D\right)=0$ (see [30]); hence, the above formula holds for $B \subset D^{c}$ (we put $P_{D}(x, z)=0$ for $z \in \partial D$ ).

By $G$ we denote the potential kernel of $X$, that is,

$$
G(x)=\int_{0}^{\infty} p_{t}(x) d t
$$

which is finite on $\mathbb{R}^{d} \backslash\{0\}$ since $d \geqslant 2$ and the global weak upper scaling condition for $\psi$ holds. For $x \in \mathbb{R}^{d} \backslash\{0\}$, we write

$$
U(x)=\frac{V^{2}(|x|)}{|x|^{d}} .
$$

We note that, by (2.5)), $U(x)$ is radially non-increasing. In [115], Theorem 3 and Section 4 , it was proved that $G(x) \approx U(x)$ for $x \neq 0$. Let

$$
r(y, z)=\delta_{D}(y) \vee \delta_{D}(z) \vee|y-z| .
$$

LEMma 3.3. Let $D$ be a bounded open $C^{1,1}$ set. Then

$$
G_{D}(y, z) \approx U(y-z) \frac{V\left(\delta_{D}(y)\right) V\left(\delta_{D}(z)\right)}{V^{2}(r(y, z))}, \quad y, z \in \mathbb{R}^{d},
$$

where the comparability constant depends only on $\psi$ and the distortion of $D$.

P r o o f. Taking the estimates of $p_{D}(t, x, y)$ (see [4], Proposition 4.4 and Theorem 4.5) and integrating them against time (see [I]], the proof of Theorem 7.3), we get

$$
G_{D}(y, z) \approx U(y-z)\left(\frac{V\left(\delta_{D}(y)\right) V\left(\delta_{D}(z)\right)}{V^{2}(|y-z|)} \wedge 1\right),
$$

where the comparability constant depends on $\psi$ only through the scaling characteristics and the distortion of $D$. Since $V$ is non-decreasing, we have

$$
\frac{V\left(\delta_{D}(y)\right) V\left(\delta_{D}(z)\right)}{V^{2}(r(y, z))} \leqslant \frac{V\left(\delta_{D}(y)\right) V\left(\delta_{D}(z)\right)}{V^{2}(|y-z|)} \wedge 1 .
$$

By the symmetry of $G_{D}(x, y)$, we may assume that $\delta_{D}(y) \leqslant \delta_{D}(z)$. If $r(y, z)=$ $|y-z|$, then

$$
\frac{V\left(\delta_{D}(y)\right) V\left(\delta_{D}(z)\right)}{V^{2}(|y-z|)} \wedge 1=\frac{V\left(\delta_{D}(y)\right) V\left(\delta_{D}(z)\right)}{V^{2}(r(y, z))} .
$$


Let $r(y, z)=\delta_{D}(z)$. If $\delta_{D}(y) \geqslant \delta_{D}(z) / 2$, then

$$
\frac{V\left(\delta_{D}(y)\right) V\left(\delta_{D}(z)\right)}{V^{2}(r(y, z))} \geqslant \frac{V\left(\delta_{D}(y)\right)}{V\left(2 \delta_{D}(y)\right)} \geqslant \frac{1}{2} \geqslant \frac{1}{2}\left(\frac{V\left(\delta_{D}(y)\right) V\left(\delta_{D}(z)\right)}{V^{2}(|y-z|)} \wedge 1\right) .
$$

If $\delta_{D}(y)<\delta_{D}(z) / 2$, then $r(y, z)=\delta_{D}(z)<2|y-z|$. Hence, by (2.5), we have, for $y, z \in D$,

$$
\frac{V\left(\delta_{D}(y)\right) V\left(\delta_{D}(z)\right)}{V^{2}(|y-z|)} \wedge 1 \leqslant \frac{V\left(\delta_{D}(y)\right) V\left(\delta_{D}(z)\right)}{V^{2}(r(y, z) / 2)} \leqslant 4 \frac{V\left(\delta_{D}(y)\right) V\left(\delta_{D}(z)\right)}{V^{2}(r(y, z))}
$$

The following result is the so-called $3 G$-theorem (see [6]).

Proposition 3.1. Let $D$ be a bounded open $C^{1,1}$ set at scale $r>0$. There is a constant $C_{2}=C_{2}(d, \psi, \operatorname{diam}(D) / r)$ such that

$$
\frac{G_{D}(x, z) G_{D}(z, y)}{G_{D}(x, y)} \leqslant C_{2} V\left(\delta_{D}(z)\right)\left(\frac{G_{D}(x, z)}{V\left(\delta_{D}(x)\right)} \vee \frac{G_{D}(z, y)}{V\left(\delta_{D}(y)\right)}\right)
$$

Proof. Let $\mathcal{G}(x, y)=U(y-x) / V^{2}(r(x, y))$. Then

$$
\mathcal{G}(x, z) \wedge \mathcal{G}(z, y) \leqslant c(d) \mathcal{G}(x, y)
$$

Indeed, assume that $|y-z| \leqslant|x-z|$; then $|x-y| \leqslant 2|x-z|$ and

$$
r(x, y) \leqslant \delta_{D}(x)+|x-y| \leqslant 3 r(x, z) .
$$

By the monotonicity of $U, V$ and (2.5) we obtain

$$
\mathcal{G}(x, z) \leqslant \frac{U((x-y) / 2)}{V^{2}(r(x, y) / 3)} \leqslant 3^{2} 2^{d} \mathcal{G}(x, y) .
$$

By Lemma B.3, $\mathcal{G}_{D}(x, y) \approx G_{D}(x, y) /\left(V\left(\delta_{D}(x)\right) V\left(\delta_{D}(y)\right)\right)$. Hence, by (B.6),

$$
\begin{aligned}
\frac{G_{D}(x, z) G_{D}(z, y)}{G_{D}(x, y)} & \approx V^{2}(\delta(z)) \frac{\mathcal{G}(x, z) \mathcal{G}(z, y)}{\mathcal{G}(x, y)} \leqslant c V^{2}(\delta(z))(\mathcal{G}(x, z) \vee \mathcal{G}(z, y)) \\
& \approx V\left(\delta_{D}(z)\right)\left(\frac{G_{D}(x, z)}{V\left(\delta_{D}(x)\right)} \vee \frac{G_{D}(z, y)}{V\left(\delta_{D}(y)\right)}\right) .
\end{aligned}
$$

The next lemma is crucial in our consideration. The proof is based on the proof of Lemma 9 in [6]. Nevertheless, we give the details, because here we can see how the weak scaling condition is used.

LEMmA 3.4. Let us assume that $0<r_{0}<\infty$ and $\operatorname{diam}(D) \leqslant r_{0}$. Then it follows that $G_{D}(y, z) /\left[\delta_{D}(z) \wedge|y-z|\right]$ is uniformly in y integrable against $|b(z)| d z$. 
Pro of. By Lemma 3.3, it is enough to prove the uniform integrability of

$$
H(y, z)=U(y-z) \frac{V\left(\delta_{D}(y)\right) V\left(\delta_{D}(z)\right)}{V^{2}(r(y, z))} \frac{\delta_{D}(z) \vee|y-z|}{|y-z| \delta_{D}(z)} .
$$

Let $A_{R}(y)=\{z \in D: H(y, z)>R\}$. We will show that

$$
\lim _{R \rightarrow \infty} \sup _{y \in D} \int_{A_{R}(y)} H(y, z)|b(z)| d z=0 .
$$

Let $\underline{c}_{2}=\underline{c}_{2}(\operatorname{diam}(D))$ be such that

$$
V(\eta r) \leqslant \underline{c}_{2} \eta^{\underline{\alpha}_{1} / 2} V(r), \quad \eta<1, r<\operatorname{diam}(D)
$$

(see Remark [.]). We recall that $\underline{\alpha}_{1}>1$. For $r>0$, we put

$$
K_{r}=\sup _{x \in \mathbb{R}^{d}} \int_{B(x, r)}|b(y)| \frac{U(x-y)}{|x-y|} d y .
$$

By (2.II), $K_{r}<\infty$ and $K_{r} \downarrow 0$ as $r \downarrow 0$. Since $U$ is a radial decreasing function, we may write $U(r)=U(x)$ for all $|x|=r$, and we have

$$
\int_{B(x, r)}|b(z)| d z \leqslant \frac{r}{U(r)} \int_{B(x, r)} \frac{U(x-z)}{|x-z|}|b(z)| d z \leqslant K_{r} \frac{r}{U(r)}, \quad x \in \mathbb{R}^{d}, r>0 .
$$

Let $m \geqslant 2$ be such that $\delta_{D}(y) \leqslant m \delta_{D}(z)$; then, by (3.7),

$$
\begin{aligned}
& H(y, z) \frac{|y-z|}{U(y-z)} \leqslant \frac{V\left(\frac{\delta_{D}(y)}{r(y, z)} r(y, z)\right) V\left(\frac{\delta_{D}(z)}{r(y, z)} r(y, z)\right)}{V(r(y, z))^{2}} \frac{r(y, z)}{\delta_{D}(z)} \\
& \leqslant \underline{c}_{2}^{2} \frac{\delta_{D}(y)^{2} / 2}{r(y, z)^{\underline{\alpha}_{1}-1} \delta_{D}(z)^{1-\underline{\alpha}_{1} / 2}} \leqslant \underline{c}_{2}^{2}\left(\frac{\delta_{D}(y)}{\delta_{D}(z)}\right)^{1-\underline{\alpha}_{1} / 2} \leqslant \underline{c}_{2}^{2} m^{1-\underline{\alpha}_{1} / 2} .
\end{aligned}
$$

By (3.7), we also have

$$
\frac{U(y-z)}{|y-z|}=\frac{V^{2}(y-z)}{|y-z|^{d+1}} \leqslant \underline{c}_{2}^{2} \frac{|y-z|^{\underline{\alpha}_{1}}}{\operatorname{diam}(D) \underline{\alpha}_{1}} V^{2}(\operatorname{diam}(D)) .
$$

Hence, the relation (B.9) yields $A_{R}(y) \subset\left\{z \in D:|y-z|<c R^{-1 /\left(d+1-\underline{\alpha}_{1}\right)}\right\}$, where $c=c\left(m,\left(\operatorname{diam}(D), \underline{\alpha}_{1}\right)\right)$ is some constant.

Let $D_{r}=\left\{x \in D: \delta_{D}(x) \geqslant r\right\}$. If $R \rightarrow \infty$, then, uniformly in $y$,

$$
\int_{A_{R}(y) \cap D_{\delta_{D}(y) / m}} H(y, z)|b(z)| d z \leqslant \underline{c}_{2}^{2} m^{1-\underline{\alpha}_{1} / 2} K_{c R^{-1 /\left(d+1-\underline{\alpha}_{1}\right)}} \rightarrow 0 .
$$


For $y \in D, k, n \geqslant 0$ and $m \geqslant 2$, we consider

$$
W_{n, k}^{m}(y)=\left\{z \in D: \frac{\delta_{D}(y)}{m 2^{n+1}}<\delta_{D}(z) \leqslant \frac{\delta_{D}(y)}{m 2^{n}}, k<\frac{|y-z|}{\delta_{D}(y)} \leqslant k+1\right\} .
$$

$W_{n, k}^{m}(y)$ may be covered by $c_{1}(k+1)^{d-2} m^{d-1} 2^{n(d-1)}$ balls of radii $\frac{\delta_{D}(y)}{m 2^{n}}$, thus

$$
\begin{aligned}
& \int_{W_{n, k}^{m}(y)}|b(z)| d z \\
& \leqslant c_{1}(k+1)^{d-2} m^{d-1} 2^{n(d-1)} \sup _{x \in \mathbb{R}^{d}} \int_{B\left(x, \delta_{D}(y) /\left(m 2^{n}\right)\right)}|b(z)| d z \\
& \leqslant c_{1} K_{\delta_{D}(y) /\left(m 2^{n}\right)}(k+1)^{d-2} m^{d-1} 2^{n(d-1)}\left(\frac{\delta_{D}(y)}{m 2^{n}}\right)^{d+1} V^{-2}\left(\frac{\delta_{D}(y)}{m 2^{n}}\right) \\
& =c_{1} K_{\delta_{D}(y) /\left(m 2^{n}\right)}(k+1)^{d-2} m^{-2} 2^{-2 n} \delta_{D}(y)^{d+1} V^{-2}\left(\frac{\delta_{D}(y)}{m 2^{n}}\right) .
\end{aligned}
$$

For $z \in W_{n, k}^{m}(y)$, we have $\delta_{D}(y) \geqslant 2 \delta_{D}(z)$, hence we get $|y-z| \geqslant \delta_{D}(y) / 2$ and $|y-z| \geqslant \delta_{D}(z)$. Therefore,

$$
H(y, z) \leqslant \frac{V\left(\delta_{D}(y)\right) V\left(\delta_{D}(z)\right)}{|y-z|^{d} \delta_{D}(z)}, \quad z \in W_{n, k}^{m}(y)
$$

and we obtain

$$
\begin{aligned}
& \int_{A_{R}(y) \backslash D_{\delta_{D}(y) / m}} H(y, z)|b(z)| d z \leqslant \sum_{n=0}^{\infty} \sum_{k=0}^{\infty} \int_{W_{n, k}^{m}(y)} \frac{V\left(\delta_{D}(y)\right) V\left(\delta_{D}(z)\right)}{|y-z|^{d} \delta_{D}(z)}|b(z)| d z \\
& \leqslant \sum_{n=0}^{\infty} \sum_{k=0}^{\infty} \frac{V\left(\delta_{D}(y)\right) V\left(\delta_{D}(y) /\left(m 2^{n}\right)\right) m 2^{n+1}}{\left((k+1) \delta_{D}(y) / 2\right)^{d} \delta_{D}(y)} \int_{W_{n, k}^{m}(y)}|b(z)| d z \\
& \leqslant c_{2} K_{\delta_{D}(y) / m} \sum_{n=0}^{\infty} \sum_{k=0}^{\infty}(k+1)^{-2} m^{-1} 2^{-n} \frac{V\left(\delta_{D}(y)\right)}{V\left(\delta_{D}(y) /\left(m 2^{n}\right)\right)} \\
& \leqslant c_{3} K_{\delta_{D}(y) / m} \sum_{n=0}^{\infty} \sum_{k=0}^{\infty}(k+1)^{-2} m^{\bar{\alpha} / 2-1} 2^{n(\bar{\alpha} / 2-1)} \leqslant c_{4} m^{\bar{\alpha} / 2-1} K_{\delta_{D}(y) / m .}
\end{aligned}
$$

Let $\varepsilon>0$. We choose $m$ and $R$ so large that $c_{4} m^{\alpha / 2-1} K_{\operatorname{diam}(D) / m}<\varepsilon / 2$ and

$$
\sup _{y \in D} \int_{D_{\delta_{D}(y) / m} \cap A_{R}(y)} H(y, z)|b(z)| d z<\varepsilon / 2 .
$$

This completes the proof.

LEMMA 3.5. If $f \in \mathcal{K}_{d}^{\nabla}$, then

$$
\nabla_{y} \int_{D} G_{D}(y, z) f(z) d z=\int_{D} \nabla_{y} G_{D}(y, z) f(z) d z, \quad y \in D .
$$


Proof. Fix $y \in D$, and let $0<h<\delta_{D}(y) / 2$ and $h_{d}=(0, \ldots, 0, h) \in \mathbb{R}^{d}$. Then

$$
\begin{aligned}
\frac{\left|G_{D}\left(y+h_{d}, z\right)-G_{D}(y, z)\right|}{h} & =\frac{1}{h}\left|\int_{0}^{1} \frac{d}{d s} G_{D}\left(y+s h_{d}, z\right) d s\right| \\
=\left|\int_{0}^{1} \frac{\partial}{\partial y_{d}} G_{D}\left(y+s h_{d}, z\right) d s\right| & \leqslant c_{1} \int_{0}^{1} \frac{G_{D}\left(y+s h_{d}, z\right)}{\left|y+s h_{d}-z\right| \wedge \delta_{D}\left(y+s h_{d}\right)} d s \\
& \leqslant c_{2} \int_{0}^{1} \frac{U\left(y+s h_{d}, z\right)}{\left|y+s h_{d}-z\right|} d s .
\end{aligned}
$$

Since $f \in \mathcal{K}_{d}^{\nabla}, U\left(y+s h_{d}, z\right) /\left(\left|y+s h_{d}-z\right|\right)$ is uniformly in $h$ integrable on $(0,1) \times D$, which completes the proof (see [6], Lemma 10).

For $x, y \in D$, we let

$$
\kappa(x, y)=\int_{D}|b(z)| \frac{G_{D}(x, z) G_{D}(z, y)}{G_{D}(x, y)\left(\delta_{D}(z) \wedge|y-z|\right)} d z,
$$

(3.14) $\widehat{\kappa}(x, y)=\int_{D}|b(z)| \frac{G_{D}(x, z) G_{D}(z, y)\left(\delta_{D}(x) \wedge|x-y|\right)}{G_{D}(x, y)\left(\delta_{D}(z) \wedge|y-z|\right)\left(\delta_{D}(x) \wedge|x-z|\right)} d z$.

Lemma 3.6. Let $\lambda<\infty, r<1$. There is $C_{3}=C_{3}(d, \psi, b, \lambda, r)$ such that if $D$ is $C^{1,1}, \operatorname{diam}(D) / r_{0}(D) \leqslant \lambda$ and $\operatorname{diam}(D) \leqslant r$, then $\kappa(x, y) \leqslant C_{3}, \widehat{\kappa}(x, y)$ $\leqslant 2 C_{3}$ for $x, y \in D$, and $C_{3}(d, \psi, b, \lambda, r) \rightarrow 0$ as $r \rightarrow 0$.

P r o of. By Lemma 3.3 and (2.7), we have

$$
\begin{aligned}
\frac{V\left(\delta_{D}(z)\right)}{V\left(\delta_{D}(x)\right)} G_{D}(x, z) & \approx \frac{V^{2}\left(\delta_{D}(z)\right)}{V^{2}(r(x, z))} U(x-z) \\
& \leqslant c\left(\frac{\delta_{D}(z)}{r(x, z)}\right)^{\underline{\alpha}}{ }_{1} U(x-z) \leqslant C\left(\delta_{D}(z) \wedge|x-z|\right) \frac{U(x-z)}{|x-z|} .
\end{aligned}
$$

By Proposition B.1, we obtain

$$
\begin{aligned}
& \frac{G_{D}(x, z) G_{D}(z, y)}{G_{D}(x, y)} \leqslant C_{2} V\left(\delta_{D}(z)\right)\left(\frac{G_{D}(x, z)}{V\left(\delta_{D}(x)\right)} \vee \frac{G_{D}(z, y)}{V\left(\delta_{D}(y)\right)}\right) \\
\leqslant & c C_{2}\left(\left(\delta_{D}(z) \wedge|x-z|\right) \frac{U(x-z)}{|x-z|}\right) \vee\left(\left(\delta_{D}(z) \wedge|y-z|\right) \frac{U(y-z)}{|y-z|}\right) \\
= & C C_{2}\left(\delta_{D}(z) \wedge|x-z| \wedge|y-z|\right)\left(\frac{U(x-z)}{|x-z|} \vee \frac{U(y-z)}{|y-z|}\right) .
\end{aligned}
$$


Hence,

$$
\frac{G_{D}(x, z) G_{D}(z, y)}{G_{D}(x, y)\left(\delta_{D}(z) \wedge|y-z| \wedge|x-z|\right)} \leqslant C C_{2}\left(\frac{U(x-z)}{|x-z|} \vee \frac{U(y-z)}{|y-z|}\right) .
$$

By (B.8) and the observation that $\lim _{r \rightarrow 0} K_{r}=0$, we have the statement for $\kappa$. The rest of the proof is the same as that of Lemma 11 in [6], so we omit it.

3.2. Green function of $\tilde{\mathcal{L}}$. We will consider analogous objects to the ones considered in the previous section. We define the Green function and the Green operator of $\tilde{\mathcal{L}}=\mathcal{L}+b \nabla$ on $D$ :

$$
\begin{gathered}
\tilde{G}_{D}(x, y)=\int_{0}^{\infty} \tilde{p}_{D}(t, x, y) d t, \quad x, y \in \mathbb{R}^{d}, \\
\tilde{G}_{D} \phi(x)=\int_{\mathbb{R}^{d}} \tilde{G}_{D}(x, y) \phi(y) d y, \quad \phi \in C^{c}\left(\mathbb{R}^{d}\right) .
\end{gathered}
$$

From the properties of $\tilde{p}_{D}(t, x, y)$ we get $\tilde{G}_{D}(x, y)=0$ if $x \in D^{c}$ or $y \in D^{c}$.

By (2.13), we have

$$
\lim _{t \rightarrow 0} \frac{\tilde{p}(t, x, y)}{t}=\lim _{t \rightarrow 0} \frac{p(t, x, y)}{t}=\nu(y-x) .
$$

Thus, the intensity of jumps of the canonical process $X_{t}$ is the same as that of $\tilde{X}_{t}$. Accordingly, we obtain the following description.

LEMmA 3.7. The $\tilde{\mathbb{P}}^{x}$-distribution of $\left(\tau_{D}, X_{\tau_{D}}\right)$ on $(0, \infty) \times(\bar{D})^{c}$ has density

$$
\int_{D} \tilde{p}_{D}(u, x, y) \nu(z-y) d y, \quad u>0, z \in(\bar{D})^{c} .
$$

We define the Poisson kernel of $D$ for $\tilde{\mathcal{L}}$,

$$
\tilde{P}_{D}(x, y)=\int_{D} \tilde{G}_{D}(x, z) \nu(y-z) d z, \quad x \in D, y \in D^{c} .
$$

By (3.16), (B.18) and (B.17), we have

$$
\tilde{\mathbb{P}}^{x}\left(X_{\tau_{D}} \in A\right)=\int_{A} \tilde{P}_{D}(x, y) d y
$$

if $A \subset(\bar{D})^{c}$. For the case of $A \subset \partial D$, we refer the reader to Lemma $4 . \mathcal{D}$. and

LEMMA 3.8. $\tilde{G}_{D}(x, y)$ is continuous for $x \neq y, \tilde{G}_{D}(x, x)=\infty$ for $x \in D$,

$$
\tilde{G}_{D}(x, y) \leqslant C_{4} U(x-y), \quad x, y \in \mathbb{R}^{d},
$$

where $C_{4}=C_{4}(d, \psi, \operatorname{diam}(D))$. 
Since the proof of the lemma is the same as the proof of Lemma 7 in [6], we omit it.

For $x \neq y$, we let

$$
G_{1}(x, y)=\int_{D} G_{D}(x, z) b(z) \cdot \nabla_{z} G_{D}(z, y) d z .
$$

By Lemma B.6,

$$
\left|G_{1}(x, y)\right| \leqslant C_{0} G_{D}(x, y) \int_{D} \frac{|b(z)| G_{D}(x, z) G_{D}(z, y)}{G_{D}(x, y)\left(\delta_{D}(z) \wedge|y-z|\right)} d z \leqslant C_{0} C_{3} G(x, y) .
$$

For $f \in \mathcal{K}_{d}^{\nabla}$, we have

$$
\begin{array}{r}
\int_{D} G_{D}(x, y) \int_{D}|b(z)| \frac{G_{D}(x, z) G_{D}(z, y)}{G_{D}(x, y)\left(\delta_{D}(z) \wedge|y-z|\right)} d z|f(y)| d y \\
\leqslant C_{3} \int_{D} G_{D}(x, y)|f(y)| d y<\infty .
\end{array}
$$

Hence, by Lemma B.5, (B.3) and Fubini's theorem,

$$
G_{D} b \nabla G_{D} f(x)=\int_{D} G_{D}(x, z) \int_{D} b(z) \cdot \nabla G_{D}(z, y) f(y) d y d z=\int_{D} G_{1}(x, y) f(y) d y .
$$

Let us note that the linear map $f \mapsto b \nabla G_{D} f$ preserves $\mathcal{K}_{d}^{\nabla}$ because $\nabla G_{D} f$ is a bounded function, see Lemma 3.4 for $b$ equal to $f$.

The next lemma results from integrating (2.26) against time.

Lemma 3.9. For all $\varphi \in C_{c}^{\infty}(D)$ and $x \in D$, we have

$$
\int_{D} \tilde{G}_{D}(x, z) \tilde{\mathcal{L}} \varphi(z) d z=\int_{D} \tilde{G}_{D}(x, z)(\mathcal{L} \varphi(z)+b(z) \cdot \nabla \varphi(z)) d z=-\varphi(x) .
$$

For every $x \in D$, let us define the function

$$
f_{x}(y)=\tilde{G}_{D}(x, y)-G_{D}(x, y)-\int_{D} \tilde{G}(x, z) b(z) \cdot \nabla_{z} G_{D}(z, y) d z .
$$

We can notice that $f_{x}(y)=0$ for $y \in \bar{D}^{c}$.

LEMMA 3.10. The function $f_{x}(y)$ is well defined on $\mathbb{R}^{d} \backslash\{x\}$, integrable on $\mathbb{R}^{d}$ and bounded on $\mathbb{R}^{d} \backslash B(x, r)$ for $r>0$.

Proof. Let us fix $y \neq x$ and $0<\rho \leqslant \min \left\{|x-y| / 2, \delta_{D}(x) / 2\right\}$. By Lemma 3.8 and $(B .3)$,

$$
\int_{D}\left|\tilde{G}_{D}(x, z) b(z) \cdot \nabla_{z} G_{D}(z, y)\right| d z \leqslant C_{4} C_{0} \int_{D} U(x-z)|b(z)| \frac{\left|G_{D}(z, y)\right|}{\delta_{D}(z) \wedge|z-y|} d z .
$$


Let $D=D_{1} \cup D_{2}$, where $D_{1}=B(x, \rho / 2)^{c} \cap D$ and $D_{2}=B(x, \rho / 2)$. By the monotonicity of $U$ and Lemma B.4,

$$
\begin{aligned}
\int_{D_{1}} U(x-z)|b(z)| & \frac{\left|G_{D}(z, y)\right|}{\delta_{D}(z) \wedge|z-y|} d z \\
& \leqslant U\left(\frac{\rho}{2}\right) \int_{D}|b(z)| \frac{\left|G_{D}(z, y)\right|}{\delta_{D}(z) \wedge|z-y|} d z \leqslant c_{1} U\left(\frac{\rho}{2}\right)
\end{aligned}
$$

for every $y \in D$. Since $b \in \mathcal{K}_{d}^{\nabla}$,

$$
\int_{D_{2}} U(x-z)|b(z)| \frac{G_{D}(z, y)}{\delta_{D}(z) \wedge|z-y|} d z \leqslant \frac{C_{4} U(\rho)}{\rho} \int_{D} U(x-z)|b(z)| d z \leqslant c_{2} \frac{U(\rho)}{\rho} .
$$

It implies that (B.23) is finite for every $y \neq x$ and bounded on $\mathbb{R}^{d} \backslash B(x, r)$ for every $r>0$.

It remains to show the integrability of $f_{x}$. Let $r=\delta_{D}(x) / 4$ and $B=B(x, 2 r)$. We put $M_{r}=\left(2 c_{1} U(r / 2)+c_{2} U(r) / r\right)|D|$. By (3.24) and (B.25),

$$
\begin{aligned}
& \int_{D} \int_{D}\left|\tilde{G}_{D}(x, z) b(z) \cdot \nabla_{z} G_{D}(z, y)\right| d z d y \\
& \leqslant M_{r}+\int_{B} \int_{B}\left|\tilde{G}_{D}(x, z) b(z) \cdot \nabla_{z} G_{D}(z, y)\right| d z d y \\
& \leqslant M_{r}+C_{0}^{2} \int_{B} \int_{B} U(x-z)|b(z)| \frac{G_{D}(z, y)}{\delta_{D}(z) \wedge|z-y|} d z d y \\
& \leqslant M_{r}+C_{0}^{2} \int_{B} \int_{B} U(x-z)|b(z)| \frac{U(z-y)}{|z-y|} d z d y \\
& \leqslant M_{r}+C_{0}^{2} \int_{D} U(x-z)|b(z)| \int_{B(z, \operatorname{diam}(D))} \frac{U(z-y)}{|z-y|} d y d z \\
& \leqslant M_{r}+c_{D} \int_{D} U(x-z)|b(z)| d z,
\end{aligned}
$$

which is finite since $b \in \mathcal{K}_{d}^{\nabla}$.

THEOREM 3.1. Let $x, y \in \mathbb{R}^{d}, x \neq y$. We have

$$
\tilde{G}_{D}(x, y)=G_{D}(x, y)+\int_{D} \tilde{G}_{D}(x, z) b(z) \cdot \nabla_{z} G_{D}(z, y) d z .
$$

Pr o of. For $x \notin D, G_{D}(x, \cdot) \equiv 0$ and (B.26) holds true. We fix $x \in D$. Let $g \in C_{c}^{\infty}(B(0,1))$ be a symmetric function such that $g \geqslant 0$ and $\int g(x) d x=1$. Let $r_{x}=\delta_{D}(x) / 3>\delta>0$ and $g_{\delta}(x)=\delta^{-d} g(x / \delta)$. Set

$$
D_{+\delta}=\{x: \operatorname{dist}(x, D)<\delta\} \quad \text { and } \quad D_{-\delta}=\{x \in D: \operatorname{dist}(x, \partial D)>\delta\} .
$$


We consider $u_{\delta, x}=g_{\delta} * f_{x} \in C_{c}^{\infty}\left(D_{+\delta}\right)$. Let $\varphi \in C_{c}^{\infty}\left(D_{-\delta}\right)$; then $g_{\delta} * \varphi \in C_{c}^{\infty}(D)$. By Lemma B.9,

$$
\left\langle g_{\delta} * f_{x}, \mathcal{L} \varphi\right\rangle=\left\langle f_{x}, g_{\delta} * \mathcal{L} \varphi\right\rangle=\left\langle f_{x}, \mathcal{L}\left(g_{\delta} * \varphi\right)\right\rangle=0 .
$$

So $u_{\delta, x}$ is weak $\mathcal{L}$-harmonic on $D_{-\delta}$. Since $u_{\delta, x} \in \mathcal{D}(\mathcal{L})$, by [9], Theorem 2.7, we get $u_{\delta, x}(y)=\mathbb{E}^{y} u_{\delta, x}\left(X_{\tau_{U}}\right)$ for every $\bar{U} \subset D_{-\delta}$. Since $\delta<r_{x}$, for every $y \in \mathbb{R}^{d}$ we have

$$
\left|u_{\delta, x}(y)\right| \leqslant \mathbb{E}^{y}\left|u_{\delta, x}\left(X_{\tau_{B\left(x, 2 r_{x}\right)}}\right)\right| \leqslant\left\|f_{x} \mathbb{1}_{B^{c}\left(x, r_{x}\right)}\right\|_{\infty}:=M .
$$

Since $\left|u_{\delta, x}(y)\right| \stackrel{\delta \rightarrow 0}{\longrightarrow}\left|f_{x}(y)\right|$ a.s., we obtain $\left|f_{x}(y)\right| \leqslant\left\|f_{x} \mathbb{1}_{B^{c}\left(x, r_{x}\right)}\right\|_{\infty}$ a.s. Since $f_{x}$ is continuous, $f_{x}$ is bounded on $\mathbb{R}^{d}$.

Let $\left\{U_{n}\right\}_{n \in \mathbb{N}}$ be a family of sets such that $U_{n} \nearrow D_{-\delta}$. By the quasi-left continuity of $X_{t}$,

$$
\begin{aligned}
& \left|u_{\delta, x}(y)\right|=\left|\lim _{n \rightarrow \infty} \mathbb{E}^{y} u_{\delta, x}\left(X_{\tau_{U_{n}}}\right)\right|=\left|\mathbb{E}^{y} \lim _{n \rightarrow \infty} u_{\delta, x}\left(X_{\tau_{U_{n}}}\right)\right|=\left|\mathbb{E}^{y} u_{\delta, x}\left(X_{\tau_{D_{-}}}\right)\right| \\
= & \left|\mathbb{E}^{y}\left(u_{\delta, x}\left(X_{\tau_{D_{-}}}\right), X_{\tau_{D_{-}}} \in D_{+\delta} \backslash D_{-\delta}\right)\right| \leqslant M \mathbb{P}^{y}\left(X_{\tau_{D_{-\delta}}} \in D_{+\delta} \backslash D_{-\delta}\right) .
\end{aligned}
$$

So $\left|u_{\delta, x}(y)\right| \leqslant M \mathbb{P}^{y}\left(X_{\tau_{D_{-}}} \in D_{+\delta} \backslash D_{-\delta}\right)$, and with $\delta \rightarrow 0$ we finally obtain

$$
\left|f_{x}(y)\right| \leqslant M \mathbb{P}^{y}\left(X_{\tau_{D}} \in \partial D\right)=0,
$$

which completes the proof.

Let $G_{0}(x, y)=G_{D}(x, y)$. We inductively define

$$
G_{n}(x, y)=\int_{D} G_{n-1}(x, z) b(z) \cdot \nabla_{z} G_{D}(z, y) d z, \quad x \neq y \in D, n=1,2, \ldots
$$

By Lemmas 3.5 and [3.6, Fubini's theorem and induction, we also have

$$
G_{n}(x, y)=\int G_{D}(x, z) b(z) \cdot \nabla_{z} G_{n-1}(z, y) d z, \quad x \neq y \in D, n=2,3, \ldots
$$

We end this section with the estimates of $\tilde{G}_{D}(x, y)$ for small sets $D$.

LEMMA 3.11. Let $d \geqslant 2, b \in \mathcal{K}_{d}^{\nabla}$ and $\lambda>0$. There is $\varepsilon=\varepsilon(d, \psi, b, \lambda)>0$ such that if $\operatorname{diam}(D) / r_{0}(D) \leqslant \lambda$ and $\operatorname{diam}(D) \leqslant \varepsilon$, then

$$
\frac{2}{3} G_{D}(x, y) \leqslant \tilde{G}_{D}(x, y) \leqslant \frac{4}{3} G_{D}(x, y), \quad x, y \in \mathbb{R}^{d} .
$$

Pro of. We follow the arguments from [6]. We present only the main steps of the proof, the details are left to the reader. 
Let $x \neq y$. Iterating (B.26), by (B.28) we obtain, for $n=0,1, \ldots$,

$$
\begin{aligned}
\tilde{G}_{D}(x, y) & =G_{D}(x, y)+\int \tilde{G}_{D}(x, z) b(z) \cdot \nabla_{z} G_{D}(z, y) d z \\
& =\sum_{k=0}^{n} G_{k}(x, y)+\int \tilde{G}_{D}(x, z) b(z) \cdot \nabla_{z} G_{n}(z, y) d z .
\end{aligned}
$$

Let $\lambda>0$. We note that the constant $C_{3}$ from Lemma 3.6 may be arbitrarily small if $\operatorname{diam}(D) / r_{0}(D) \leqslant \lambda$ and $r_{0}(D)$ is small enough. Hence, we may choose $\varepsilon=\varepsilon(d, \psi, b, \lambda)>0$ such that $C_{0} C_{3}<1 / 4$. By (ㅍ.2), (3.20), Lemma 3.6 and induction,

$$
\begin{gathered}
\left|G_{n}(x, y)\right| \leqslant \int_{D}\left|G_{n-1}(x, z)\right||b(z)|\left|\nabla_{z} G_{D}(z, y)\right| d z \\
\leqslant\left(C_{0} C_{3}\right)^{n-1} \int_{D} G_{D}(x, z)|b(z)|\left|\nabla_{z} G_{D}(z, y)\right| d z \leqslant 4^{-n} G_{D}(x, y), \\
\left|\nabla_{x} G_{n}(x, y)\right| \leqslant 2^{-n} C_{0} \frac{G_{D}(x, y)}{\delta_{D}(x) \wedge|x-y|}
\end{gathered}
$$

for $n=0,1,2, \ldots$ Now, we have $\tilde{G}_{D}(x, y)=\sum_{n=0}^{\infty} G_{n}(x, y)$. Indeed, by (3.32), the remainder in (B.30) is bounded by

$$
2^{-n} C_{0} \int_{D} U(x-z)|b(z)| \frac{G_{D}(z, y)}{\delta_{D}(z) \wedge|y-z|} d z \rightarrow 0 \quad \text { as } n \rightarrow \infty .
$$

The integral is finite because of Lemma B.4. Thus, by (B.3I),

$$
\begin{aligned}
& \tilde{G}_{D}(x, y) \leqslant \sum_{n=0}^{\infty} G_{n}(x, y) \leqslant \sum_{n=0}^{\infty} 4^{-n} G_{D}(x, y)=\frac{4}{3} G_{D}(x, y), \\
& \tilde{G}_{D}(x, y) \geqslant G_{D}(x, y)-\sum_{n=1}^{\infty} 4^{-n} G_{D}(x, y)=\frac{2}{3} G_{D}(x, y) .
\end{aligned}
$$

\section{PROOF OF THEOREM 1.1}

Using the comparability of $G_{D}$ and $\tilde{G}_{D}$ for small $C^{1,1}$ sets and repeating the arguments from [6], we obtain estimates of the Poisson kernel and Harnack principles. The proofs are almost identical to the ones from [6]. Nevertheless, due to the references we use, we present them below.

By the Ikeda-Watanabe formula, we get

$$
\tilde{\mathbb{P}}^{x}\left(X_{\tau_{D}} \in A\right) \approx \mathbb{P}^{x}\left(X_{\tau_{D}} \in A\right), \quad x \in D, A \subset(\bar{D})^{c},
$$

for a sufficiently small $\operatorname{diam}(D)$ and bounded distortion. The next lemma says that the process $\tilde{X}_{t}$ does not hit the boundary of our general $C^{1,1}$ open set $D$ at the moment of the first exit from $D$. 
LEMMA 4.1. For every $x \in D$, we have $\tilde{\mathbb{P}}^{x}\left(X_{\tau_{D}} \in \partial D\right)=0$.

Proof. Let $u(x)=\tilde{\mathbb{P}}^{x}\left(X_{\tau_{D}} \in \partial D\right), x \in \mathbb{R}^{d}$. We claim that there exists $c=$ $c(d, \psi, D, b)>0$ such that $u(x)<1-c$ for $x \in D$. Indeed, we consider small $\varepsilon>0$, $x \in D, r=\varepsilon \operatorname{dist}\left(x, D^{c}\right)$, the ball $B=B(x, r / 2) \subset D$, and the ball $B^{\prime} \subset(\bar{D})^{c}$ with radius and distance to $B$ comparable with $r$. By (4.1), (2.13) and Lemma B.7,

$$
\tilde{\mathbb{P}}^{x}\left(X_{\tau_{D}} \notin \partial D\right) \geqslant \tilde{\mathbb{P}}^{x}\left(X_{\tau_{B(x, r / 2)}} \in B^{\prime}\right) \approx \mathbb{P}^{x}\left(X_{\tau_{B(x, r / 2)}} \in B^{\prime}\right) \geqslant c,
$$

where in the last inequality we used ([5.5),$(2.10)$, (2.5) and [27]. Furthermore, let $D_{n}=\left\{y \in D: \operatorname{dist}\left(y, D^{c}\right)>1 / n\right\}, n=1,2, \ldots$ We consider $n$ such that $B(x, r / 2) \subset D_{n}$. We have $\tilde{\mathbb{P}}^{x}\left(X_{\tau_{D_{n}}} \in \bar{D}\right) \leqslant 1-\tilde{\mathbb{P}}^{x}\left(X_{\tau_{B}} \in B^{\prime}\right) \leqslant 1-c$, as before. Let $C=\sup \{u(y): y \in D\}$. We have $u(x)=\tilde{\mathbb{E}}^{x}\left\{u\left(X_{\tau_{D_{n}}}\right): X_{\tau_{D_{n}}} \in \bar{D}\right\} \leqslant$ $C(1-c)$, hence $C \leqslant C(1-c)$, and so $C=0$.

In the context of Lemma $3 . \mathrm{W}$, the $\tilde{\mathbb{P}}^{x}$ distribution of $X_{\tau_{D}}$ is absolutely continuous with respect to the Lebesgue measure and has the density function

$$
\tilde{P}_{D}(x, y) \approx P_{D}(x, y), \quad y \in D^{c},
$$

provided $x \in D$. This follows from (B.19) and Lemma 4.1. For clarity,

$$
\tilde{\mathbb{P}}^{x}\left(X_{\tau_{S}} \in A\right) \approx \mathbb{P}^{x}\left(X_{\tau_{S}} \in A\right), \quad x \in S, A \subset S^{c} .
$$

LEMMA 4.2 (Harnack inequality for $\tilde{\mathcal{L}}$ ). Let $x, y \in \mathbb{R}^{d}, 0<s<1$ and $k \in \mathbb{N}$ satisfy $|x-y| \leqslant 2^{k}$ s. Let $u$ be non-negative in $\mathbb{R}^{d}$ and $\tilde{\mathcal{L}}$-harmonic in $B(x, s) \cup$ $B(y, s)$. There is $C_{5}=C_{5}(d, \psi, b)$ such that

$$
C_{5}^{-1} 2^{-k(d+\bar{\alpha})} u(x) \leqslant u(y) \leqslant C_{5} 2^{k(d+\bar{\alpha})} u(x) .
$$

Proof. We may assume that $s \leqslant 1 \wedge \varepsilon / 2$, with $\varepsilon$ of Lemma B.Dl. Let $f(z)=$ $u(z)$ for $z \in B(y, 2 s / 3)^{c}$ and $f(z)=\int_{B(y, 2 s / 3)^{c}} u(v) P_{B(y, 2 s / 3)}(z, v) d v$ for $z \in$ $B(y, 2 s / 3)$, so that $f$ is non-negative in $\mathbb{R}^{d}$ and $\mathcal{L}$-harmonic in $B(y, 2 s / 3)$. Let $z \in B(y, s / 2)$. By (4.3)),

$$
u(z)=\tilde{\mathbb{E}}^{z} u\left(X\left(\tau_{B(y, 2 s / 3)}\right)\right)=\int_{B(y, 2 s / 3)^{c}} u(v) \tilde{P}_{B(y, 2 s / 3)}(z, v) d v \approx f(z) .
$$

The Harnack inequality for $\mathcal{L}$ (see [15]) implies $u(y) \approx u(z)$, where the comparability constant depends on $\psi, d, b$. The standard chain rule provides $u(x) \approx u(y)$ for $|x-y|<3 / 2 s$. Therefore, we assume that $|x-y| \geqslant 3 s / 2$. For $z \in B(y, s / 2)$ and $w \in B(x, s / 2)$ we have $|w-z| \leqslant|x-y|+|y-z|+|w-x| \leqslant 2^{k} s+s \leqslant$ $2^{k+1} s$. Hence, by the Ikeda-Watanabe formula, (2.10) and [27],

$$
\begin{aligned}
P_{B(x, s / 2)}(x, z) & =\int_{B(x, s / 2)} G_{B(x, s / 2)}(x, w) \nu(|w-z|) d w \geqslant \mathbb{E}^{x} \tau_{B(x, s / 2)} \nu\left(2^{k+1} s\right) \\
& \approx \frac{\psi\left(1 /\left(2^{k+1} s\right)\right)}{\left(2^{k+1} s\right)^{d} \psi(2 / s)} \geqslant \frac{1}{\left(2^{k+1} s\right)^{d} \bar{C} 2^{\bar{\alpha}(k+2)}} \geqslant \frac{1}{2^{k(d+\bar{\alpha})} \bar{C} 2^{d+2 \bar{\alpha}}} s^{-d} .
\end{aligned}
$$


Since $\tilde{P}_{B(x, s / 2)} \approx P_{B(x, s / 2)}$, by the first part of the proof we obtain

$$
\begin{aligned}
& u(x)=\int_{B(x, s / 2)^{c}} \tilde{P}_{B(x, s / 2)}(x, z) u(z) d z \geqslant \int_{B(y, s / 2)} \tilde{P}_{B(x, s / 2)}(x, z) u(z) d z \\
\approx & \int_{B(y, s / 2)} P_{B(x, s / 2)}(x, z) u(z) d z \geqslant \frac{c|B(y, s / 2)|}{2^{k(d+\bar{\alpha})} s^{d} 2^{d+2 \bar{\alpha}}} u(y)=C_{5} 2^{-k(d+\bar{\alpha})} u(y) .
\end{aligned}
$$

By symmetry, $u(x) \approx u(y)$.

We obtain also the boundary Harnack principle for $\mathcal{L}$ and general $C^{1,1}$ sets $D$.

LEMMA 4.3 (boundary Harnack principle). Let $z \in \partial D, 0<r \leqslant r_{0}(D)$, and $0<p<1$. If $\tilde{u}, \tilde{v}$ are non-negative in $\mathbb{R}^{d}$, regular $\tilde{\mathcal{L}}$-harmonic in $D \cap B(z, r)$, vanish on $D^{c} \cap B(z, r)$ and satisfy $\tilde{u}\left(x_{0}\right)=\tilde{v}\left(x_{0}\right)$ for some $x_{0} \in D \cap B(z, p r)$, then

$$
C_{6}^{-1} \tilde{v}(x) \leqslant \tilde{u}(x) \leqslant C_{6} \tilde{v}(x), \quad x \in D \cap B(z, p r),
$$

with $C_{6}=C_{6}\left(d, \psi, b, p, r_{0}(D)\right)$.

Proof. In view of Lemma 4.2 we may assume that $r$ is small. Let $F=$ $F(z, r / 2) \subset B(z, r)$ be the $C^{1,1}$ domain of Lemma B.], localizing $D$ at $z$. For $x \in$ $F$ we have $\tilde{u}(x)=\int \tilde{P}_{F}(x, z) \tilde{u}(z) d z \approx u(x)$, where $u(x)=\int P_{F}(x, z) \tilde{u}(z) d z$. Similarly, $\tilde{v}(x) \approx v(x)=\int P_{F}(x, z) \tilde{v}(z) d z$. Since $\tilde{u}\left(x_{0}\right)=\tilde{v}\left(x_{0}\right)$, we have $u\left(x_{0}\right)$ $\approx v\left(x_{0}\right)$. By [2]], Theorem 2.18, $u(x) \approx v(x)$ provided $x \in D \cap B(z, r / 8)$. We use Lemma 4.2 for the full range $x \in D \cap B(z, p r)$.

Now, we have all the tools necessary to prove the main result of our paper. Since in the proof we follow the idea from [6], we only give its basic steps (for details see [6], the proof of Theorem 1).

Pro of of The or e m ‥]. By (B.26) and (B.3), we have the estimate

$$
\tilde{G}_{D}(x, y) \leqslant G_{D}(x, y)+C_{0} \int_{D} \frac{\tilde{G}_{D}(x, z) G_{D}(z, y)}{\delta_{D}(z) \wedge|y-z|}|b(z)| d z, \quad x, y \in D .
$$

We consider $\eta<1$, say $\eta=1 / 2$. By Lemma 3.4 and the uniform integrability in Lemma 3.6 (see (B.15)), there is a constant $r>0$ so small that

$$
\begin{array}{r}
\int_{D_{r}} \frac{G_{D}(z, y)}{\delta_{D}(z) \wedge|y-z|}|b(z)| d z<\frac{\eta}{C_{0}}, \quad y \in D, \\
\int_{D_{r}} \frac{G_{D}(x, z) G_{D}(z, y)}{G_{D}(x, y)\left(\delta_{D}(z) \wedge|y-z|\right)}|b(z)| d z<\frac{\eta}{C_{0}}, \quad y \in D .
\end{array}
$$


Here, $D_{r}=\left\{z \in D: \delta_{D}(z) \leqslant r\right\}$. We put

$$
\rho=\left[\varepsilon \wedge r_{0}(D) \wedge r\right] / 16,
$$

with $\varepsilon=\varepsilon(d, \psi, b, 2 / \kappa)$ of Lemma B.W1, see also Lemma B.D.

To prove ([L.3) we will consider $x$ and $y$ in the partitions of $D \times D$.

First, we consider $y$ far from the boundary of $D$, say $\delta_{D}(y) \geqslant \rho / 4$.

- For $|x-y| \leqslant \rho / 8, G_{D}(x, y) \approx G_{B}(x, y) \approx U(x-y) \approx \tilde{G}_{D}(x, y)$ (we use Lemmas [.3. 3. [1], (3.8).

- If $\rho / 8<\delta_{D}(x)$, we use the Harnack inequalities for $\mathcal{L}$ and $\tilde{\mathcal{L}}$.

- For $\delta_{D}(x)<\rho / 8$, we use the boundary Harnack principle (see Lemma 4.3 and [21], Theorem 2.18).

Next, suppose that $\delta_{D}(y) \leqslant \rho / 4$. Here, the difficulty lies in the fact that $\tilde{G}_{D}$ is non-symmetric.

In the proof of lower bounds we consider two cases: $x$ close to $y$, and $x$ far away from $y$.

- In the case $|x-y| \leqslant \rho$, we locally approximate $D$ by a small $C^{1,1}$ set $F$ such that $\delta_{D}(x)=\delta_{F}(x)$ and $\delta_{D}(y)=\delta_{F}(y)$ (see [6], Lemma 1). Then $\tilde{G}_{D}(x, y)$ $\geqslant \tilde{G}_{F}(x, y) \approx G_{F}(x, y) \approx G_{D}(x, y)$ (see Lemma B.3).

- For $|x-y|>\rho$ and $\delta_{D}(x) \geqslant \rho / 4$ we use the Harnack inequalities. For $\delta_{D}(x) \leqslant \rho / 4$ we use the boundary Harnack principle.

In the next step, we prove the upper bound in ([1.3) for $\delta_{D}(x) \geqslant \rho / 4$. We have already proved that, for $z \in D \backslash D^{r}$,

$$
c_{1}^{-1} G_{D}(x, z) \leqslant \tilde{G}_{D}(x, z) \leqslant c_{1} G_{D}(x, z) .
$$

By (3.13), Lemmas 3.6 and 3.8 , and (4.6), (4.7), we have

$$
\begin{aligned}
& \tilde{G}_{D}(x, y) \leqslant A G_{D}(x, y)+C_{0} \int_{D_{r}} \frac{\tilde{G}_{D}(x, z) G_{D}(z, y)}{|y-z| \wedge \delta_{D}(z)}|b(z)| d z, \\
& \tilde{G}_{D}(x, y) \leqslant A G_{D}(x, y)+B(x),
\end{aligned}
$$

where $A=1+c_{1} C_{0} C_{3}$ and $B(x)=\eta C_{4} U\left(\delta_{D^{r}}(x)\right)$. Now, plugging (4.10) into (4.9), and using (4.7), (4.8) and induction, we get, for $n=0,1, \ldots$,

$$
\tilde{G}_{D}(x, y) \leqslant A\left(1+\eta+\ldots+\eta^{n}\right) G_{D}(x, y)+\eta^{n} B(x) .
$$

In consequence,

$$
\tilde{G}_{D}(x, y) \leqslant \frac{A}{1-\eta} G_{D}(x, y)
$$


Finally, we prove the upper bound in ([1.3) when $\delta_{D}(x)<\rho / 4$.

- If $|x-y|>\rho$, we use the boundary Harnack principle.

- For $|x-y| \leqslant \rho$, consider the same set $F$ as above. We have

$$
\tilde{G}_{D}(x, y)=\tilde{G}_{F}(x, y)+\int_{D \backslash F} \tilde{P}_{F}(x, z) \tilde{G}_{D}(z, y) d z .
$$

By Lemma 3.11 and (4.2), $\tilde{G}_{F}(x, y) \approx G_{F}(x, y)$ and $\tilde{P}_{F}(x, z) \approx P_{F}(x, z)$. We already know that, for $|z-y|>\rho, \tilde{G}_{D}(z, y) \approx G(z, y)$. Thus,

$$
\tilde{G}_{D}(x, y) \approx G_{F}(x, y)+\int_{D \backslash F} P_{F}(x, z) G_{D}(z, y) d z=G_{D}(x, y) .
$$

The proof of Theorem $\square$ is is complete.

\section{REFERENCES}

[1] R. M. Blumenthal and R. K. Getoor, Markov Processes and Potential Theory, Pure and Applied Mathematics, Vol. 29, Academic Press, New York 1968.

[2] K. Bogdan, Y. Butko, and K. Szczypkowski, Majorization, $4 G$ Theorem and Schrödinger perturbations, J. Evol. Equ. 16 (2016), pp. 241-260.

[3] K. Bogdan, T. Grzywny, and M. Ryznar, Density and tails of unimodal convolution semigroups, J. Funct. Anal. 266 (6) (2014), pp. 3543-3571.

[4] K. Bogdan, T. Grzywny, and M. Ryznar, Dirichlet heat kernel for unimodal Lévy processes, Stochastic Process. Appl. 124 (11) (2014), pp. 3612-3650.

[5] K. Bogdan and T. Jakubowski, Estimates of heat kernel of fractional Laplacian perturbed by gradient operators, Comm. Math. Phys. 271 (1) (2007), pp. 179-198.

[6] K. Bogdan and T. Jakubowski, Estimates of the Green function for the fractional Laplacian perturbed by gradient, Potential Anal. 36 (3) (2012), pp. 455-481.

[7] K. Bogdan and T. Komorow ski, Principal eigenvalue of the fractional Laplacian with a large incompressible drift, NoDEA Nonlinear Differential Equations Appl. 21 (4) (2014), pp. $541-566$

[8] L. A. Caffarelli and A. Vasseur, Drift diffusion equations with fractional diffusion and the quasi-geostrophic equation, Ann. of Math. (2) 171 (3) (2010), pp. 1903-1930.

[9] Z.-Q. Chen, On notions of harmonicity, Proc. Amer. Math. Soc. 137 (10) (2009), pp. $3497-$ 3510 .

[10] Z.-Q. Chen, P. Kim, and R. Song, Dirichlet heat kernel estimates for fractional Laplacian with gradient perturbation, Ann. Probab. 40 (6) (2012), pp. 2483-2538.

[11] Z.-Q. Chen, P. Kim, and R. Song, Dirichlet heat kernel estimates for rotationally symmetric Lévy processes, Proc. Lond. Math. Soc. (3) 109 (1) (2014), pp. 90-120.

[12] Z.-Q. Chen, Y.-X. Ren, and T. Yang, Boundary Harnack principle and gradient estimates for fractional Laplacian perturbed by non-local operators, Potential Anal. 45 (3) (2016), pp. 509-537.

[13] K. L. Chung and Z. X. Zhao, From Brownian Motion to Schrödinger's Equation, Grundlehren Math. Wiss., Vol. 312, Springer, Berlin 1995.

[14] P. Graczyk, T. Jakubowski, and T. Luks, Martin representation and Relative Fatou Theorem for fractional Laplacian with a gradient perturbation, Positivity 17 (4) (2013), pp. $1043-1070$.

[15] T. Grzy wny, On Harnack inequality and Hölder regularity for isotropic unimodal Lévy processes, Potential Anal. 41 (1) (2014), pp. 1-29. 
[16] T. Grzywny and K. Szczypkowski, Estimates of heat kernels of non-symmetric jump processes, preprint 2016.

[17] N. Ikeda and S. Watanabe, On some relations between the harmonic measure and the Lévy measure for a certain class of Markov processes, J. Math. Kyoto Univ. 2 (1962), pp. 79-95.

[18] T. Jakubowski, On Harnack inequality for $\alpha$-stable Ornstein-Uhlenbeck processes, Math. Z. 258 (3) (2008), pp. 609-628.

[19] T. Jakubowski, Fractional Laplacian with singular drift, Studia Math. 207 (3) (2011), pp. 257-273.

[20] T. Jakubowski and K. Szczypkowski, Estimates of gradient perturbation series, J. Math. Anal. Appl. 389 (1) (2012), pp. 452-460.

[21] P. Kim, R. Song, and Z. Vondraček, Martin boundary for some symmetric Lévy processes, in: Festschrift Masatoshi Fukushima, Z.-Q. Chen, N. Jacob, M. Takeda, and T. Uemura (Eds.), World Scientific, 2015, pp. 307-342.

[22] A. Kiselev, F. Nazarov, and A. Volberg, Global well-posedness for the critical 2D dissipative quasi-geostrophic equation, Invent. Math. 167 (3) (2007), pp. 445-453.

[23] T. Kulczycki and M. Ryznar, Gradient estimates of harmonic functions and transition densities for Lévy processes, Trans. Amer. Math. Soc. 368 (1) (2016), pp. 281-318.

[24] Y. Maekawa and H. Miura, Upper bounds for fundamental solutions to non-local diffusion equations with divergence free drift, J. Funct. Anal. 264 (10) (2013), pp. 2245-2268.

[25] A. Petrosy an and C. A. Pop, Optimal regularity of solutions to the obstacle problem for the fractional Laplacian with drift, J. Funct. Anal. 268 (2) (2015), pp. 417-472.

[26] N. I. Portenko, Some perturbations of drift-type for symmetric stable processes, Random Oper. Stoch. Equ. 2 (3) (1994), pp. 211-224.

[27] W. E. Pruitt, The growth of random walks and Lévy processes, Ann. Probab. 9 (6) (1981), pp. 948-956.

[28] L. Silvestre, Hölder estimates for advection fractional-diffusion equations, Ann. Sc. Norm. Super. Pisa Cl. Sci. (5) 11 (4) (2012), pp. 843-855.

[29] L. Silvestre, V. Vicol, and A. Zlatoš, On the loss of continuity for super-critical driftdiffusion equations, Arch. Ration. Mech. Anal. 207 (3) (2013), pp. 845-877.

[30] P. Sztonyk, On harmonic measure for Lévy processes, Probab. Math. Statist. 20 (2) (2000), pp. 383-390.

[31] T. Watanabe, The isoperimetric inequality for isotropic unimodal Lévy processes, Z. Wahrsch. Verw. Gebiete 63 (4) (1983), pp. 487-499.

Tomasz Grzywny

Faculty of Pure and Applied Mathematics

Wrocław University of Science and Technology

Wybrzeże Wyspiańskiego 27

50-370 Wrocław, Poland

E-mail: tomasz.grzywny@pwr.edu.pl
Tomasz Jakubowski

Faculty of Pure and Applied Mathematics Wrocław University of Science and Technology Wybrzeże Wyspiańskiego 27 50-370 Wrocław, Poland

E-mail: tomasz.jakubowski@pwr.edu.pl

Grzegorz Żurek

Faculty of Pure and Applied Mathematics

Wrocław University of Science and Technology

Wybrzeże Wyspiańskiego 27

50-370 Wrocław, Poland

E-mail: grzegorz.zurek@pwr.edu.pl

Received on 3.6.2015;

revised version on 28.2.2016 